\title{
Thai Language Convention
}

For most Thai words, this book adheres to the phonetic transcription of the "General System of Phonetic Transcription of Thai Characters into Roman" devised by the Royal Institute, Bangkok, in 1954. In the case of a name which is widely known or which can be checked, the owner's transcription is used. The English names of certain Thai royals, such as Chulalongkorn, Bhumibol, and Vajiralongkorn, have been adopted rather than the lengthy official titles. Thai people are referred to by their first names while Westerners are referred to by their surnames. In the text and bibliography, Thai names are entered according to first names. 